\title{
Eicosapentaenoic Acid-Enriched High-Density Lipoproteins Exhibit Anti-Atherogenic Properties
}

\author{
Nobuaki Tanaka, MD, PhD; Yasuhiro Irino, PhD; Masakazu Shinohara, MD, PhD; \\ Shigeyasu Tsuda, MD; Takeshige Mori, MD; Manabu Nagao, MD, PhD; \\ Toshihiko Oshita, MD; Kenta Mori, MD, PhD; Tetsuya Hara, MD, PhD; \\ Ryuji Toh, MD, PhD; Tatsuro Ishida, MD, PhD; Ken-ichi Hirata, MD, PhD
}

\begin{abstract}
Background: It has previously been reported that oral administration of purified eicosapentaenoic acid (EPA) generates EPA-rich high-density lipoprotein (HDL) particles with a variety of anti-inflammatory properties. In this study, the mechanism underlying the anti-atherogenic effects of EPA-rich HDL using reconstituted $\mathrm{HDL}$ (rHDL) was investigated.

Methods and Results: rHDL was generated by the sodium cholate dialysis method, using apolipoprotein A-1 protein, cholesterol, and various concentrations of EPA-phosphatidylcholine (PC) or egg-PC. Increased EPA-PC contents in rHDL resulted in decreased particle size. Next, the effects of rHDL containing various amounts (0-100\% of total PC) of EPA-PC on vascular cell adhesion molecule-1 (VCAM-1) expression in human umbilical vein endothelial cells (HUVECs) was examined. Cytokine-stimulated VCAM-1 expression was inhibited in a dose-dependent manner based on the amount of EPA-PC in rHDL. Surprisingly, the incubation of HUVECs with EPA-rich $\mathrm{rHDL}$ resulted in the production of resolvin E3 (RvE3), an anti-inflammatory metabolite derived from EPA. Incubation with EPA-PC alone did not adequately induce RvE3 production, suggesting that RvE3 production requires an endothelial cell-HDL interaction. The increased anti-inflammatory effects of EPA-rich HDL may be explained by EPA itself and RvE3 production. Furthermore, the increase in EPA-PC content enhanced cholesterol efflux.
\end{abstract}

Conclusions: The EPA-enriched HDL particles exhibit cardioprotective properties via the production of anti-inflammatory lipid metabolites and the increase in cholesterol efflux.

Key Words: High-density lipoprotein (HDL); Inflammation; Lipids; Lipoproteins; Omega-3 fatty acid

$\mathbf{M}$ any epidemiological studies have shown an inverse association between high-density lipoprotein (HDL) cholesterol levels and coronary artery disease (CAD). ${ }^{1-3}$ Although HDL has multiple functions that contribute to protective effects against atherosclerosis, ${ }^{4}$ including anti-inflammatory, anti-oxidative, and anti-apoptotic properties, a major prospective effect of HDL is its ability to deliver cholesterol from the peripheral tissues to the liver for biliary excretion through a process known as reverse cholesterol transport. Emerging evidence has revealed a negative correlation between cholesterol efflux and the probability of CAD that is independent of the HDL cholesterol concentration..$^{\mathbf{5 , 6}}$ Thus, it is generally accepted that not only the quantity, but also the quality, of HDL are accurate predictors of CAD risk. ${ }^{7.8}$

\begin{abstract}
Editorial p 332
Long-chain n-3 polyunsaturated fatty acids (PUFAs), including $\alpha$-linolenic acid, eicosapentaenoic acid (EPA), and docosahexaenoic acid, possess a variety of cardioprotective properties. Supplementation with n-3 PUFA reduces triglyceride levels in both normolipidemic subjects and hypertriglyceridemic patients. ${ }^{9}$ In Japan, high-dose administration of EPA ethyl ester decreased the number of major coronary events in hypercholesterolemic patients (Japan EPA Lipid Intervention Study (JELIS)). ${ }^{10,11}$ High-dose EPA reduced the levels of triglyceride and inflammatory markers, such as lipoprotein-associated phospholipase A2 and high-sensitive reactive protein $\mathrm{C}$, and improved other
\end{abstract}

Received March 15, 2017; revised manuscript received May 24, 2017; accepted May 25, 2017; released online June 23 , 2017 Time for primary review: 23 days

Division of Cardiovascular Medicine (N.T., S.T., T.M., M.N., T.O., K.M., T.H., T.I., K.H.), Division of Evidence-based Laboratory Medicine (Y.I., R.T., K.H.), The Integrated Center for Mass Spectrometry (Y.I., M.S.), Division of Epidemiology (M.S.), Kobe University Graduate School of Medicine, Kobe, Japan

Y.I. and M.S. contributed equally to this work.

Mailing address: Yasuhiro Irino, PhD, Division of Evidence-based Laboratory Medicine, Kobe University Graduate School of Medicine, 7-5-1 Kusunoki-cho, Chuo-ku, Kobe 650-0017, Japan. E-mail: irino@med.kobe-u.ac.jp and Masakazu Shinohara, $\mathrm{MD}, \mathrm{PhD}$, Division of Epidemiology, Kobe University Graduate School of Medicine, 7-5-1 Kusunoki-cho, Chuo-ku, Kobe 650-0017, Japan. E-mail: mashino@med.kobe-u.ac.jp

ISSN-1346-9843 All rights are reserved to the Japanese Circulation Society. For permissions, please e-mail: cj@j-circ.or.jp 
lipid parameters, including non-HDL cholesterol and ApoB in the Multicenter, Placebo-controlled, Randomized, Double-blind, 12-week Study With an Open-label Extension (MARINE) and Effect of AMR101 (Icosapent Ethyl) on Triglyceride Levels in Patients on Statins With High Triglyceride Levels (ANCHOR) studies. ${ }^{12-14}$ In addition, we have recently demonstrated that administration of high-dose EPA enhances the anti-inflammatory properties of HDL in Japanese patients with dyslipidemia. ${ }^{15}$ Evidence favors the possibility that EPA plays crucial roles in the prevention of cardiovascular diseases; ${ }^{\mathbf{1 6}}$ however, the molecular mechanisms by which EPA exerts its antiatherosclerotic effects remain unclear.

In this study, to determine the effects of EPA on HDL function, we generated reconstituted HDL (rHDL) particles containing various amounts of EPA and examined their effects on anti-atherosclerotic properties, such as antiinflammatory functions and cholesterol efflux.

\section{Methods}

\section{Materials}

Human apolipoprotein A-1 (apoA-1), 1,2-Dieicosapentaenoyl-sn-glycero-3-phosphocholine, and 1,2-Diacyl-snglycero-3-phosphocholine from egg yolk were purchased from Sigma-Aldrich (St. Louis, MO, USA). Tumor necrosis factor- $\alpha(\mathrm{TNF}-\alpha)$ was purchased from WAKO (Osaka, Japan). Antibodies against 5-lipoxygenase, 12-lipoxygenase, and 15-lipoxygenase were purchased from Santa Cruz Biotechnology (Dallas, TX, USA).

\section{Preparation of rHDL}

The rHDL particles were prepared using a previously described sodium cholate dialysis method. ${ }^{17}$ Briefly, the required amounts of phosphatidylcholine (PC) and cholesterol were mixed and dried under a $\mathrm{N}_{2}$ gas stream. The dried mixture was solved in tris(hydroxymethyl)aminomethane (Tris)-buffered saline (TBS; $8.2 \mathrm{mmol} / \mathrm{L}$ Tris- $\mathrm{HCl}$, $150 \mathrm{mmol} / \mathrm{L} \mathrm{NaCl}, \mathrm{pH} 8.0$ ) and supplemented with $19 \mathrm{mmol} / \mathrm{L}$ sodium deoxycholate until the solution was clear. ApoA-1 was added to the solution to make a final PC:cholesterol:apoA-1 molar ratio of 160:8:1. The mixture was incubated at $37^{\circ} \mathrm{C}$ for $1 \mathrm{~h}$ and dialyzed against TBS for 3 days to remove sodium deoxycholate. Protein concentration was measured by using the Bradford protein assay.

The samples were subjected to non-denaturing 4-20\% gradient polyacryamide gel (Bio-Rad, Hercules, CA, USA) electrophoresis and stained with Coomassie Brilliant Blue to visualize the rHDL particles. Particle size was assigned by a comparison with protein standards using a High

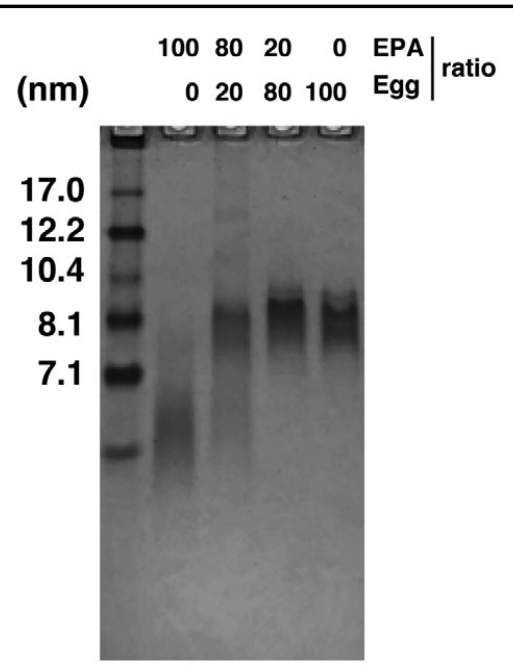

Figure 1. Native polyacrylamide gel electrophoresis (PAGE) analysis of reconstituted high-density lipoprotein ( $\mathrm{rHDL}$ ) prepared at different eicosapentaenoic acid-phosphatidylcholine (EPA-PC) molar ratios in a 4-20\% polyacrylamide gel. Standard proteins of known hydrodynamic diameters were used for this analysis. Samples $(1.0 \mu \mathrm{g}$ proteins) were separated by non-denaturing gel electrophoresis and stained with Coomassie Brilliant Blue.

Molecular Weight Calibration Kit (GE Healthcare, Buckinghamshire, England).

\section{Vascular Cell Adhesion Molecule-1 Expression}

Vascular adhesion molecule-1 (VCAM-1) expression was examined by using a previously described method. ${ }^{15}$ Briefly, human umbilical vein endothelial cells (HUVECs) were plated in serum-deprived RPMI1640 medium supplemented with $1 \%$ fetal bovine serum (FBS). HUVECs were incubated with RPMI1640 supplemented with 1\% FBS, $100 \mu \mathrm{g} / \mathrm{mL} \mathrm{rHDL}$ and $10 \mathrm{ng} / \mathrm{mL}$ TNF- $\alpha$ for $4 \mathrm{~h}$. VCAM-1 expression was measured by using real-time polymerase chain reaction (PCR). All data were normalized to $\beta$-actin expression.

\section{Cholesterol Efflux Assay}

The cholesterol efflux was determined by using a commercially available assay kit (Abcam, Cambridge, England) according to the manufacturer's instructions. Briefly, J774.1 cells were pre-incubated for $16 \mathrm{~h}$ with the labeling reagent

\begin{tabular}{|lcccc|}
\hline \multicolumn{5}{|c|}{ Table. Fatty Acid Compositions of the rHDL Used in This Study, as Examined Using GC-MS } \\
Fatty acid & $\begin{array}{c}\text { EPA-HDL } \\
\mathbf{( 1 0 0 )}\end{array}$ & $\begin{array}{c}\text { EPA-HDL } \\
\mathbf{( 8 0 )}\end{array}$ & $\begin{array}{c}\text { EPA-HDL } \\
(\mathbf{2 0})\end{array}$ & Egg HDL \\
Palmitate $(\mu \mathrm{mol} / \mathrm{L})$ & 25.5 & 373.0 & $1,192.7$ & $1,602.3$ \\
Stearate $(\mu \mathrm{mol} / \mathrm{L})$ & 13.6 & 129.9 & 400.5 & 507.9 \\
Oleate $(\mu \mathrm{mol} / \mathrm{L})$ & 20.1 & 355.1 & $1,228.9$ & $1,529.3$ \\
Linoleate $(\mu \mathrm{mol} / \mathrm{L})$ & 5.9 & 169.1 & 581.2 & 715.0 \\
Arachidonate $(\mu \mathrm{mol} / \mathrm{L})$ & 11.7 & 48.0 & 125.2 & 138.9 \\
Eicosapentaenoate $(\mu \mathrm{mol} / \mathrm{L})$ & $3,007.2$ & $3,502.8$ & 611.1 & 6.2 \\
\hline
\end{tabular}

The rHDL preparation contained EPA-PC:egg-PC ratios of 100:0 (EPA-HDL (100)) to 0:100 (egg HDL). EPA, eicosapentaenoic acid; GC-MS, gas chromatography-mass spectrometry; rHDL, reconstituted high-density lipoprotein. 

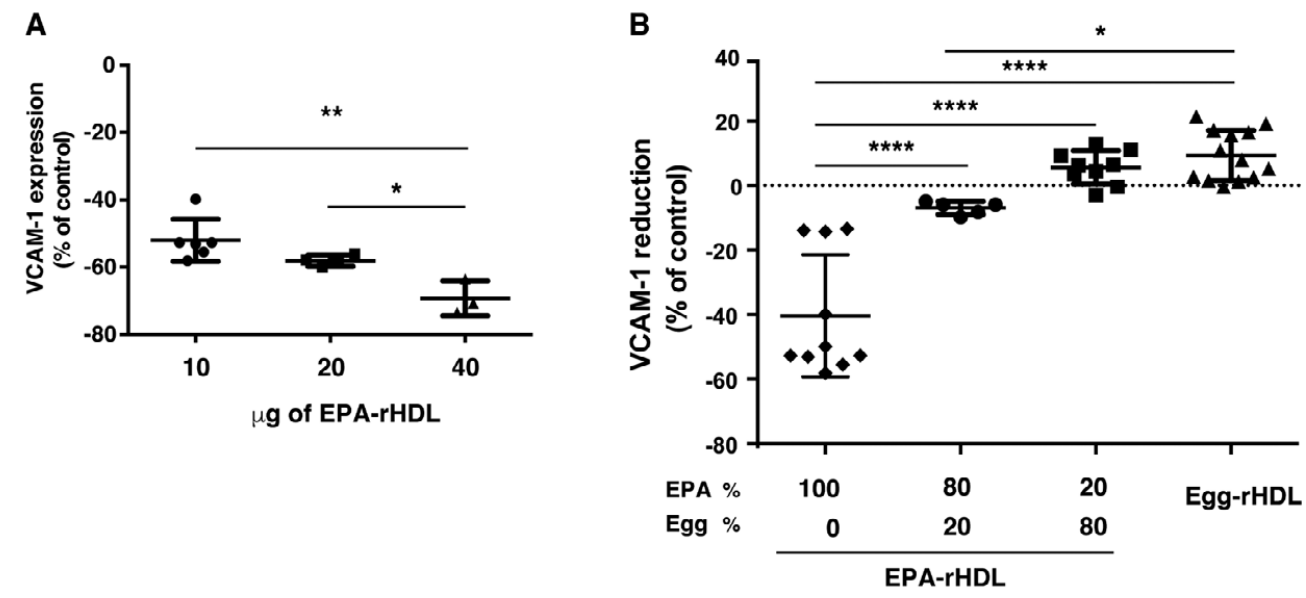

Figure 2. EPA-rich rHDL inhibited cytokine-stimulated vascular adhesion molecule-1 (VCAM-1) expression. (A) Concentrationdependent rHDL-induced suppression of tumor necrosis factor (TNF)- $a$-induced VCAM-1 expression. Human umbilical vein endothelial cells (HUVECs) were pre-incubated with the indicated concentrations of rHDL containing EPA-PC:egg-PC ratios of 100:0 overnight and stimulated with TNF- $a$ for $4 \mathrm{~h}$. The relative VCAM-1 expression levels were analyzed by using real-time polymerase chain reaction. ${ }^{*} \mathrm{P}<0.05,{ }^{* *} \mathrm{P}<0.01$. P-values were determined by analysis of variance (ANOVA) with Tukey's multiple comparisons post-hoc test. (B) Inhibitory effects of EPA-PC concentration in rHDL on HUVECs. Increase in EPA-PC contents in rHDL enhanced the suppression of VCAM-1 expression. rHDL was supplemented at $10 \mu \mathrm{g}$ protein $/ \mathrm{mL} .{ }^{*} \mathrm{P}<0.05,{ }^{* * * *} \mathrm{P}<0.0001$. P-values were determined by using an ANOVA with Tukey's multiple comparisons post-hoc test. Abbreviations as in Figure 1.

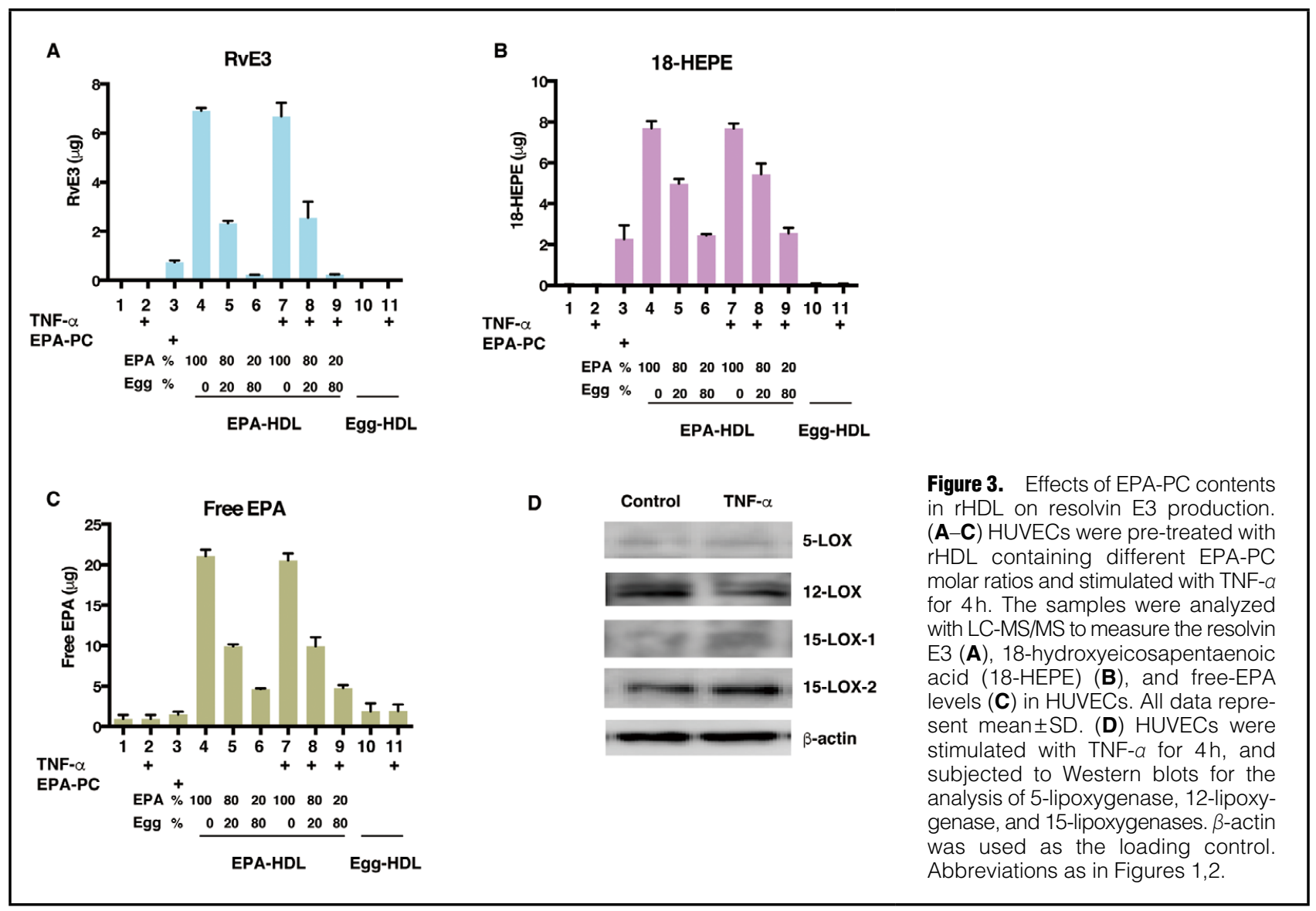


and equilibration buffer (provided in the kit). The cells were washed with the medium and incubated with rHDL for $4 \mathrm{~h}$. Media were removed and the cells were lysed in cell lysis buffer (provided in the kit). Cholesterol efflux into the medium was expressed as a percentage using the following formula: fluorescence intensity (FI) in the medium/(FI in the medium + FI in the cells $) \times 100$.

\section{Fatty Acid Profiling}

Fatty acids were purified from HDL and methylated by using a commercially available kit (Nacalai Tesque, Kyoto, Japan) according to the manufacturer's protocol. The concentrations of methylated fatty acids were measured using gas chromatography-mass spectrometry (GC-MS). The GC-MS conditions used for the measurements in this study are described in a previous study. ${ }^{18}$

\section{Measurement of Lipid Mediators}

Incubations were terminated by adding a $2 \times$ volume of ice-cold methanol. Then, the deuterated internal standards, leukotriene $\mathrm{B}_{4}-\mathrm{d}_{4}\left(\mathrm{~d}_{4}-\mathrm{LTB}_{4}\right)$, d8-5-hydroxyeicosatetraenoic acid (d8-5-HETE), d4-prostaglandin $\mathrm{E}_{2}\left(\mathrm{~d}_{4}-\mathrm{PGE}_{2}\right)$, and $\mathrm{d}_{5}$-resolvin D2 (d5-RvD2), which represented each chromatographic region of the identified LMs, were added to the samples $(500 \mathrm{pg}$ each) to facilitate quantification. Samples underwent solid phase extraction (SPE) on C18 columns and were subjected to liquid chromatographymass spectrometry (LC-MS/MS). The system consisted of a Qtrap 6500 (Sciex, Framingham, MA, USA), equipped with a Shimadzu LC-30AD HPLC system. A ZORBAX Eclipse Plus C18 column ( $100 \mathrm{~mm} \times 4.6 \mathrm{~mm}, 3.5 \mu \mathrm{m}$; Agilent Technologies, Santa Clara, CA, USA) was used with a gradient of methanol/water/acetic acid from 55:45:0.01 $(\mathrm{v} / \mathrm{v} / \mathrm{v})$ to $98: 2: 0.01$ at a flow rate of $0.4 \mathrm{~mL} / \mathrm{min}$. A multiple reaction monitoring (MRM) method was developed for the signature ion pairs Q1 (parent ion)/Q3 (characteristic fragment ion) of each molecule to monitor and quantify the levels of targeted LMs. The LMs were identified using published criteria, such as LC retention time, specific fragmentation patterns, and diagnostic fragmentation ions. The samples were quantified based on the peak area of the MRM chromatograph, and linear calibration curves were obtained with authentic standards for each compound. ${ }^{19}$

\section{Statistical Analysis}

A one-way analysis of variance (ANOVA) with Tukey's post-hoc test was used to analyze the comparisons as indicated. All statistical analyses were performed using GraphPad Prism software.

\section{Results}

\section{Preparation and Characterization of rHDL}

Discoidal rHDL was prepared by mixing apoA-1 and free cholesterol to create different amounts of EPA-PC. EggPC, free cholesterol, and apoA-1 produced rHDL particles with diameters of $\sim 10 \mathrm{~nm}$ (Figure 1). In contrast, the increasing amounts of EPA-PC in rHDL gradually reduced the rHDL particle size, suggesting that EPA-PC is responsible for the HDL particle size.

We measured the fatty acid compositions of PC in rHDL using GC-MS analysis. The amount of EPA was increased in proportion to the EPA-PC contents in rHDL, while the amounts of other fatty acids, including palmitate, stearate, oleate, linoleate, and arachidonate, were reduced
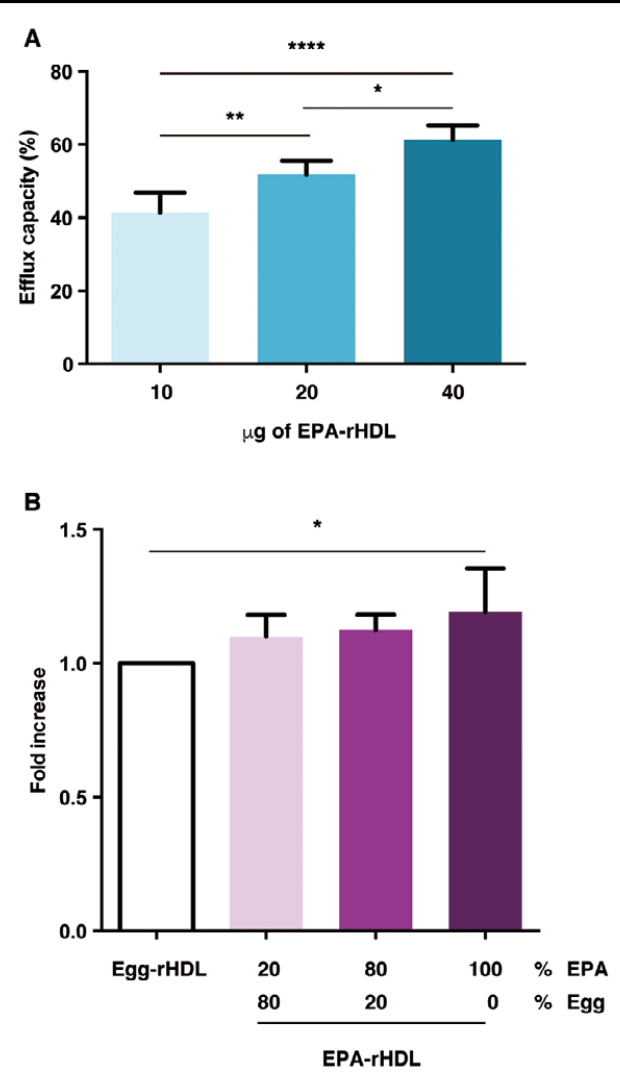

Figure 4. Effects of EPA-PC contents in rHDL on cholesterol efflux. (A) Dose-dependent effects of rHDL on cholesterol efflux. Cholesterol efflux from macrophages was measured over $4 \mathrm{~h}$ following stimulation with the rHDL particles containing EPA-PC:egg-PC ratios of $100: 0$. ${ }^{*} P<0.05$, ${ }^{*} P<0.01$, ${ }^{* * *} P<0.0001$. P-values were determined by ANOVA with Tukey's multiple comparisons post-hoc test. (B) Cholesterol efflux from macrophages in response to rHDL particles. Increase in EPA-PC contents in rHDL enhanced the cholestero efflux capacity of rHDL. rHDL was supplemented at $100 \mu \mathrm{g}$ protein $/ \mathrm{mL}$. ${ }^{*} \mathrm{P}<0.05$. P-values were determined by ANOVA with Tukey's multiple comparisons post-hoc test. Abbreviations as in Figures 1,2.

(Table).

\section{Anti-Inflammatory Properties of rHDL Containing EPA}

We next examined the effects of EPA on the anti-inflammatory properties of rHDL. First, HUVECs were preincubated with various concentrations of rHDL containing EPA-PC:egg-PC ratios of 100:0 and stimulated with TNF- $\alpha$ for 4 h. VCAM-1 expression was dose-dependently inhibited by rHDL (Figure 2A). Next, we used rHDL particles with various EPA-PC contents to examine whether rHDL enriched in EPA-PC exhibited increased antiinflammatory activity. As shown in Figure 2B, rHDL highly enriched with EPA efficiently blocked TNF- $\alpha$ stimulated VCAM-1 expression, whereas rHDL with a low amount of EPA-PC was less effective.

\section{Role of Resolvin E3 in the Anti-Inflammatory Effects}

Based on growing evidence, specialized pro-resolving mediators (SPMs), particularly the resolvin family derived from long-chain n-3 fatty acids, such as EPA and DHA, ${ }^{20-22}$ 
actively stimulate the resolution of inflammation. Among the resolvins, E-series resolvins are known as EPA metabolites. We hypothesized that the SPMs were involved in the anti-inflammatory properties of EPA-rich rHDL. Treatment with EPA-rich rHDL dramatically increased the amount of resolvin E3 (RvE3) in HUVECs, depending on the EPA content in rHDL (Figure 3A, columns 4-6), while TNF- $\alpha$ stimulation had no effect on RvE3 production (Figure $\mathbf{3 A}$, columns 7-9). Egg HDL supplementation did not increase RvE3 production because of the low amount of EPA in egg HDL (Table). Surprisingly, the addition of liposomes containing EPA-PC failed to effectively generate RvE3 compared to EPA-rHDL (Figure 3A, column 3), suggesting that HDL particles are required for efficient RvE3 production from endothelial cells, and that liposomes are not sufficient to support resolvin production.

The intermediate metabolite, 18-hydroxyeicosapentaenoic acid (18-HEPE), is derived from EPA and further converted to RvE3 by 15 -lipooxygenase. Consistent with the RvE3 levels, the levels of 18-HEPE and free EPA also increased in response to EPA-rHDL supplementation (Figure 3B,3C). Finally, we determined whether HUVECs possessed the lipoxygenases required for RvE3 synthesis by using Western blotting. All lipoxygenases were expressed in HUVECs, and there was no difference between TNF- $\alpha$-stimulated and unstimulated cells (Figure 3D).

\section{Effects of the Phospholipid Composition of rHDL on Cholesterol Efflux}

Based on the evidence presented above, we concluded that EPA-enriched rHDL has anti-inflammatory properties. A major function of HDL is to protect against atherosclerosis via cholesterol efflux. ${ }^{5}$ Thus, we explored the roles of EPA$\mathrm{PC}$ in $\mathrm{rHDL}$ in cholesterol efflux. First, we confirmed that cholesterol efflux from macrophages was dependent on the rHDL dose (Figure 4A). Furthermore, increasing EPA-PC concentrations enhanced cholesterol efflux ability (Figure 4B), suggesting a crucial role for EPA-PC in rHDL in cholesterol efflux activity.

\section{Discussion}

According to the study results, the phospholipid composition of rHDL altered the cholesterol efflux and anti-inflammatory effects of HDL. For the first time, the E-series resolvins were shown to be involved in the anti-inflammatory effects of HDL, and the production of the E-series resolvins depended on the interaction between HDL and endothelial cells. Moreover, the increasing EPA-PC contents in rHDL decreased the particle size, suggesting that EPA-rich rHDL forms saddle-shaped, minimal surface particles in high-fluidity membranes, which is consistent with the findings from a previous study. ${ }^{23}$

There are possible mechanisms by which EPA-PC decreases rHDL particle size. Because EPA-PC increases membrane fluidity, the lipid bilayer in EPA-rich rHDL forms a saddle surface, which results in smaller rHDL particles. It is also possible that conformational changes in ApoA-1 or the number of ApoA-1 molecules, depending on EPA-PC amounts, may influence HDL particle size. It will be important to further investigate the role of EPA-PC contents on HDL particle size.

The unsaturated fatty acid composition of the phospholipids in HDL was shown to be associated with a significant suppression of VCAM-1 expression in several studies. ${ }^{15,24}$
In the present study, RvE3, a potent specialized proresolving mediator, ${ }^{22}$ was produced after the interaction between EPA-rich rHDL and endothelial cells. RvE3 was biosynthesized from EPA by P450 or aspirin-acetylated cyclooxygenase-2, followed by 15 -lipoxygenase activity. ${ }^{25}$ TNF- $\alpha$ stimulation had no effects on lipoxygenase expression, and EPA-rich rHDL induced RvE3 production, suggesting that RvE3 production is directly reflected by the amount of EPA.

Surprisingly, the HDL structure appeared to be necessary for the efficient conversion of EPA-PC to 18-HEPE and RvE3 by endothelial cells. Addition of EPA-PC liposomes slightly increases the intracellular amounts of EPA, 18-HEPE, and RvE3 in cells, probably through passive fusion of the plasma membrane and liposomes. ${ }^{26}$ Notably, the addition of EPA-HDL remarkably increases EPA, 18-HEPE, and RvE3 levels. Moreover, because the production of EPA, 18-HEPE, and RvE3 is proportional to the EPA content in HDL, they are generated from PC in rHDL but not in cells. Therefore, we speculate that HDL is incorporated into cells using active transport machinery. It will be worthwhile to investigate in future studies whether HDL is actively incorporated into the cells.

We previously reported that orally administrated EPA was efficiently incorporated into the HDL particles to generate EPA-rich HDL. ${ }^{15}$ In addition, it is widely recognized that anti-inflammatory effects of E-series resolvins are nearly 1,000-fold greater than those of EPA. ${ }^{22}$ Taken together, dietary EPA consumption that improves cardiovascular function may be associated with RvE3 production from the EPA-incorporated HDL.

As shown in recent studies, smaller HDL particles are highly efficient in ATP-binding cassette transporter 1-(ABCA1) dependent cholesterol efflux, ${ }^{27,28}$ providing strong evidence for the enhanced cholesterol efflux ability of EPA-rich HDL reported here. Du et al suggested that the capacity of small HDL species for ABCA1-dependent cell surface binding may be related to their ability to promote cholesterol efflux. ${ }^{28}$ Conversely, in the study by Marmillot et al, the cholesterol efflux capacity of saturated lipid-based rHDL was higher than that of rHDL incorporating unsaturated lipids. ${ }^{29}$ However, results from all studies are not easily comparable because the experimental conditions, such as cell type, rHDL concentration, and duration of incubation with rHDL and cells, varied. Notably, the phospholipid composition in rHDL affects its cholesterol efflux capacity. We cannot exclude the possibility that apoA-1 was released from rHDL and was converted to lipid-poor or lipid-free apoA-1. Further studies are required to investigate the roles of the EPA in HDL in cholesterol efflux.

\section{Study Limitation}

The oral EPA therapy in daily practices is unable to achieve the equivalent concentrations of EPA, which was observed in rHDL used in this study. However, our findings may indicate that the functions of HDL are potentially affected by its fatty acid composition in phospholipids. Further studies are required to confirm the present study findings in clinical samples.

\section{Conclusions}

The EPA content in HDL influences the size of HDL particles, cholesterol efflux, and cytokine-induced VCAM-1 
expression by producing E-series resolvins. Our findings provide novel evidence that allows us to better understand the mechanism by which dietary EPA consumption improves HDL function and reduces cardiovascular risks.

\section{Acknowledgments}

We would like to thank Emiko Yoshida for providing technical assistance.

\section{Grants}

This work was supported by a Grant-in-Aid for Scientific Research (C) to T.I. (15K09121) and a Grant-in-Aid for Scientific Research on Innovative Areas to M.S. (16H01361).

\section{Disclosure}

The authors declare no conflicts of interest.

\section{References}

1. Gordon T, Castelli WP, Hjortland MC, Kannel WB, Dawber TR. High density lipoprotein as a protective factor against coronary heart disease: The Framingham Study. Am J Med 1977; 62: $707-714$.

2. Despres JP, Lemieux I, Dagenais GR, Cantin B, Lamarche B. HDL-cholesterol as a marker of coronary heart disease risk: The Quebec cardiovascular study. Atherosclerosis 2000; 153: 263272.

3. Gotto AM Jr. Low high-density lipoprotein cholesterol as a risk factor in coronary heart disease: A working group report. Circulation 2001; 103: 2213-2218.

4. Annema W, von Eckardstein A. High-density lipoproteins: Multifunctional but vulnerable protections from atherosclerosis. Circ J 2013; 77: 2432-2448.

5. Khera AV, Cuchel M, de la Llera-Moya M, Rodrigues A, Burke MF, Jafri K, et al. Cholesterol efflux capacity, high-density lipoprotein function, and atherosclerosis. N Engl J Med 2011; 364: $127-135$.

6. Rohatgi A, Khera A, Berry JD, Givens EG, Ayers CR, Wedin $\mathrm{KE}$, et al. HDL cholesterol efflux capacity and incident cardiovascular events. $N$ Engl J Med 2014; 371: 2383-2393.

7. Salahuddin T, Natarajan B, Playford MP, Joshi AA, Teague H, Masmoudi Y, et al. Cholesterol efflux capacity in humans with psoriasis is inversely related to non-calcified burden of coronary atherosclerosis. Eur Heart J 2015; 36: 2662-2665.

8. Bhatt A, Rohatgi A. HDL cholesterol efflux capacity: Cardiovascular risk factor and potential therapeutic target. Curr Atheroscler Rep 2016; 18: 2.

9. Zuliani G, Galvani M, Leitersdorf E, Volpato S, Cavalieri M, Fellin R. The role of polyunsaturated fatty acids (PUFA) in the treatment of dyslipidemias. Curr Pharm Des 2009; 15: 4087-4093.

10. Saito Y, Yokoyama M, Origasa H, Matsuzaki M, Matsuzawa Y, Ishikawa Y, et al. Effects of EPA on coronary artery disease in hypercholesterolemic patients with multiple risk factors: Subanalysis of primary prevention cases from the Japan EPA Lipid Intervention Study (JELIS). Atherosclerosis 2008; 200: 135-140.

11. Yokoyama M, Origasa H, Matsuzaki M, Matsuzawa Y, Saito Y, Ishikawa $\mathrm{Y}$, et al. Effects of eicosapentaenoic acid on major coronary events in hypercholesterolaemic patients (JELIS): A randomised open-label, blinded endpoint analysis. Lancet 2007; 369: $1090-1098$.

12. Ballantyne CM, Bays HE, Kastelein JJ, Stein E, Isaacsohn JL, Braeckman RA, et al. Efficacy and safety of eicosapentaenoic acid ethyl ester (AMR101) therapy in statin-treated patients with persistent high triglycerides (from the ANCHOR study). Am J Cardiol 2012; 110: 984-992.

13. Bays HE, Ballantyne CM, Braeckman RA, Stirtan WG, Soni PN. Icosapent ethyl, a pure ethyl ester of eicosapentaenoic acid: Effects on circulating markers of inflammation from the MARINE and ANCHOR studies. Am J Cardiovasc Drugs 2013; 13: $37-46$.

14. Bays HE, Ballantyne CM, Kastelein JJ, Isaacsohn JL, Braeckman RA, Soni PN. Eicosapentaenoic acid ethyl ester (AMR101) therapy in patients with very high triglyceride levels (from the Multi-center, plAcebo-controlled, Randomized, doubleblINd, 12-week study with an open-label Extension [MARINE] trial). Am J Cardiol 2011; 108: 682-690.

15. Tanaka N, Ishida T, Nagao M, Mori T, Monguchi T, Sasaki M, et al. Administration of high dose eicosapentaenoic acid enhances anti-inflammatory properties of high-density lipoprotein in Japanese patients with dyslipidemia. Atherosclerosis 2014; 237: $577-583$.

16. Swanson D, Block R, Mousa SA. Omega-3 fatty acids EPA and DHA: Health benefits throughout life. Adv Nutr 2012; 3: 1-7.

17. Cavigiolio G, Shao B, Geier EG, Ren G, Heinecke JW, Oda MN. The interplay between size, morphology, stability, and functionality of high-density lipoprotein subclasses. Biochemistry 2008; 47: 4770-4779.

18. Mori K, Ishida T, Yasuda T, Hasokawa M, Monguchi T, Sasaki $M$, et al. Serum trans-fatty acid concentration is elevated in young patients with coronary artery disease in Japan. Circ J 2015; 79: 2017-2025.

19. Colas RA, Shinohara M, Dalli J, Chiang N, Serhan CN. Identification and signature profiles for pro-resolving and inflammatory lipid mediators in human tissue. Am J Physiol Cell Physiol 2014; 307: C39-C54.

20. Shinohara M, Serhan CN. Novel endogenous proresolving molecules: Essential fatty acid-derived and gaseous mediators in the resolution of inflammation. $J$ Atheroscler Thromb 2016; 23: 655-664.

21. Arita M, Bianchini F, Aliberti J, Sher A, Chiang N, Hong S, et al. Stereochemical assignment, antiinflammatory properties, and receptor for the omega-3 lipid mediator resolvin E1. J Exp Med 2005; 201: 713-722.

22. Serhan CN. Pro-resolving lipid mediators are leads for resolution physiology. Nature 2014; 510: $92-101$.

23. Miyazaki M, Nakano M, Fukuda M, Handa T. Smaller discoidal high-density lipoprotein particles form saddle surfaces, but not planar bilayers. Biochemistry 2009; 48: 7756-7763.

24. Baker PW, Rye KA, Gamble JR, Vadas MA, Barter PJ. Phospholipid composition of reconstituted high density lipoproteins influences their ability to inhibit endothelial cell adhesion molecule expression. J Lipid Res 2000; 41: 1261-1267.

25. Isobe $\mathrm{Y}$, Arita $\mathrm{M}$, Matsueda $\mathrm{S}$, Iwamoto $\mathrm{R}$, Fujihara $\mathrm{T}$, Nakanishi H, et al. Identification and structure determination of novel anti-inflammatory mediator resolvin E3, 17,18-dihydroxyeicosapentaenoic acid. J Biol Chem 2012; 287: 10525-10534.

26. Bozzuto G, Molinari A. Liposomes as nanomedical devices. Int J Nanomedicine 2015; 10: 975-999.

27. Favari E, Calabresi L, Adorni MP, Jessup W, Simonelli S, Franceschini G, et al. Small discoidal pre-betal HDL particles are efficient acceptors of cell cholesterol via ABCA1 and ABCG1. Biochemistry 2009; 48: 11067-11074.

28. Du XM, Kim MJ, Hou L, Le Goff W, Chapman MJ, Van Eck $\mathrm{M}$, et al. HDL particle size is a critical determinant of ABCA1mediated macrophage cellular cholesterol export. Circ Res 2015; 116: $1133-1142$.

29. Marmillot P, Patel S, Lakshman MR. Reverse cholesterol transport is regulated by varying fatty acyl chain saturation and sphingomyelin content in reconstituted high-density lipoproteins. Metabolism 2007; 56: 251-259. 